\title{
Scutellaria baicalensis Extracts and Flavonoids Protect Rat L6 Cells from Antimycin A-Induced Mitochondrial Dysfunction
}

\author{
A-Rang Im, ${ }^{1}$ Young-Hwa Kim, ${ }^{1}$ Md. Romij Uddin, ${ }^{1,}, 2$ Hye Won Lee, ${ }^{1}$ Seong Wook Chae, \\ Yun Hee Kim, ${ }^{1}$ Woo Suk Jung, ${ }^{1}$ Bong Ju Kang, ${ }^{1}$ Chun Sun Mun, ${ }^{3}$ and Mi-Young Lee ${ }^{1}$ \\ ${ }^{1}$ Korea Institute of Oriental Medicine, 461-24 Jeonmin-dong, Yuseong-gu, Daejeon 305-811, Republic of Korea \\ ${ }^{2}$ Department of Crop Science, Chungnam National University, Daejeon 305-764, Republic of Korea \\ ${ }^{3}$ School of Chinese Materia Medica, Beijing University of Chinese Medicine, Beijing 100102, China
}

Correspondence should be addressed to Mi-Young Lee, mylee@kiom.re.kr

Received 11 June 2012; Revised 20 July 2012; Accepted 23 July 2012

Academic Editor: Vassya Bankova

Copyright (C) 2012 A-Rang Im et al. This is an open access article distributed under the Creative Commons Attribution License, which permits unrestricted use, distribution, and reproduction in any medium, provided the original work is properly cited.

\begin{abstract}
Antimycin A (AMA) damages mitochondria by inhibiting mitochondrial electron transport and can produce reactive oxygen species (ROS). ROS formation, aging, and reduction of mitochondrial biogenesis contribute to mitochondrial dysfunction. The present study sought to investigate extracts of Scutellaria baicalensis and its flavonoids (baicalin, baicalein, and wogonin), whether they could protect mitochondria against oxidative damage. The viability of L6 cells treated with AMA increased in the presence of flavonoids and extracts of S. baicalensis. ATP production decreased in the AMA treated group, but increased by $50 \%$ in cells treated with flavonoids (except wogonin) and extracts of $S$. baicalensis compared to AMA-treated group. AMA treatment caused a significant reduction (depolarized) in mitochondrial membrane potential (MMP), whereas flavonoid treatment induced a significant increase in MMP. Mitochondrial superoxide levels increased in AMA treated cells, whereas its levels decreased when cells were treated with flavonoids or extracts of S. baicalensis. L6 cells treated with flavonoids and extracts of S. baicalensis increased their levels of protein expression compared with AMA-treated cells, especially water extracts performed the highest levels of protein expression. These results suggest that the S. baicalensis extracts and flavonoids protect against AMA-induced mitochondrial dysfunction by increasing ATP production, upregulating MMP, and enhancing mitochondrial function.
\end{abstract}

\section{Introduction}

The generation of ROS, which are products of respiration, is believed to contribute substantially to aging $[1,2]$. Oxidative stress and mitochondrial dysfunction are important factors that contribute to aging [3]. A series of protein complexes (I-IV) is embedded in the inner mitochondrial membrane generating a proton gradient known as the mitochondrial membrane potential (MMP) [4]. The mitochondrial respiratory chain is a major site of ROS production in the cell. Generations of ROS play an important role in mitochondrial dysfunction and represent putative targets of antiaging strategies [5]. Antimycin A (AMA) damages mitochondria in many cell types by inhibiting mitochondrial electron transport $[6,7]$. Complexes I and III of the mitochondrial electron transport chain are the major sites for ROS production. In mitochondria, AMA binds to the Qi site of cytochrome $c$ reductase, thereby the oxidation of ubiquinol in the electron transport chain of oxidative phosphorylation. AMA is known to cause the leakage of superoxide radicals from rat liver mitochondria [8].

The dried root of $S$. baicalensis is rich in flavonoids, containing over 30 different kinds of flavonoids. Phytochemical investigations revealed that flavonoids primarily comprise baicalin, baicalein, and wogonin. The antioxidants and anti-inflammatory flavonoids, baicalin, baicalein, and wogonin are present in abundance in this medicinal herb [9-13]. Ethanol extracts of S. baicalensis prevent oxidative damage and neuroinflammation, and aqueous extracts have oxidative effects in cultured human umbilical vein endothelial cells $[14,15]$. Baicalein, baicalin, and wogonin each affect mitochondrial function [16]. Baicalein protects 
mitochondria against oxidative damage via induction of manganese superoxide dismutase and hydrogen peroxideinduced oxidative stress in HS-SY5Y cells $[17,18]$. Baicalin also acts as a prooxidant and induces caspase- 3 activation and apoptosis via the mitochondrial pathway [19]. Despite the many studies of $S$. baicalensis extracts and flavonoids isolated from them, little is known about their effects on antimycin A-induced mitochondrial dysfunction. The goal of the present study was to increase our understanding of how and how much of these compounds mitigate the mitochondrial damaging activity of AMA towards mitochondria by assessing the protective effect from both the extracts and flavonoids (baicalein, baicalin, and wogonin) of Scutellaria baicalensis against AMA-induced L6 cells.

\section{Materials and Methods}

2.1. Materials. Baicalein, baicalin, and wogonin were purchased from Wako Pure (Tokyo, Japan). AMA and DAPI solutions were purchased from Sigma Chemical (St. Louis, MO, USA). Dulbecco's modified Eagle's medium (DMEM) and fetal bovine serum (FBS) were purchased from Gibco BRL (Grand Island, NY). CellTiter Aqueous One Solution Cell proliferation assay kit ([3-(4,5-dimethylthiazol-2-yl)-5(3-carboxymethoxyphenyl)-2-(4-sulfophenyl)-2H-tetrazolium, inner salt; MTS) was purchased from Promega Co. (Madison, WI, USA). ATP levels and MMP were determined using a Luminescence ATP detection kit (PerkinElmer, Waltham, MA, USA) and the JC-1 mitochondrial membrane potential detection kit (Biotium, Hayward, CA, USA). Mitotracker and MitoSOX were purchased from Invitrogen Molecular Probes (San Diego, CA, USA). The pAMPK primary antibody and a secondary antibody (anti-goat and rabbit) were purchased from Santa Cruz Biotechnology Inc. (Santa Cruz, CA, USA). The PGC1 $\alpha$ and SIRT1 antibodies were obtained from Abcam Ltd (Cambridge, UK).

2.2. Preparation of S. baicalensis Extracts. Aqueous extracts of S. baicalensis were prepared by sonicating the dried ground powder $(150.58 \mathrm{~g})$ that was suspended in distilled water for $2 \mathrm{~h}$. The process was repeated three times. The suspension was lyophilized, yielding $78.45 \mathrm{~g}$ of water extract. A $70 \%$ ethanol extract was prepared by sonicating the dried ground powder $(150.01 \mathrm{~g})$ suspended in $70 \%$ ethanol solvent $(\mathrm{v} / \mathrm{v} \%$ in water), and the suspension was processed as described for the aqueous extract yielding $35.28 \mathrm{~g}$.

2.3. Cell Culture. The L6 skeletal muscle cell line was purchased from the Korean Cell Line Bank (Seoul, Korea) and maintained at subconfluence at $37^{\circ} \mathrm{C}$ in a humidified atmosphere of $95 \%$ air and $5 \% \mathrm{CO}_{2}$. The cells were grown in DMEM with $10 \%$ FBS containing 100 units/mL of penicillin and $100 \mu \mathrm{g} / \mathrm{mL}$ of streptomycin.

2.4. Cell Viability and MTS Assay. L6 cells were plated at a density of $1 \times 10^{4}$ cells/well in DMEM containing $10 \%$ FBS in a 96 -well plate and were incubated at $37^{\circ} \mathrm{C}$ for $24 \mathrm{~h}$. Cells were treated with varying concentrations of $S$. baicalensis extracts in a 96-well plate after additional incubation at $37^{\circ} \mathrm{C}$. Cell viability was determined after $24 \mathrm{~h}$ by reduction of MTS to its formazan product. After removing the medium, $200 \mu \mathrm{L}$ DMEM containing MTS was added to each well, and samples were then incubated at $37^{\circ} \mathrm{C}$ for $60 \mathrm{~min}$. The absorbance of the reaction at $490 \mathrm{~nm}$ was determined using a microplate fluorometer (Molecular Devices, Sunnyvale, CA, USA). To determine whether samples could protect cells against AMA, varying concentrations of extracts were added to the plates $1 \mathrm{~h}$ after adding $100 \mu \mathrm{g} / \mathrm{mL}$ AMA. The MTS assay was performed $24 \mathrm{~h}$ later as described previously.

2.5. ATP Assays. Varying amounts of extracts were added to cells in a 96-well white plate for $1 \mathrm{~h}$ before adding $100 \mu \mathrm{g} / \mathrm{mL}$ AMA. Total cellular ATP content was determined using an ATP luminescence detection kit and a luminometer [20]. The values, compared with an internal standard, are expressed as percentages of untreated cells (control).

2.6. Mitochondrial Membrane Potential. To detect changes in MMP, JC-1 was used as an indicator of mitochondrial function. The dye 5, 5', 6, 6'-tetrachloro-1, 1', 3, 3' tetraethylbenzimidazolylcarbocyanine iodide (JC-1) fluoresces red or green, respectively, when it aggregates in healthy mitochondria with high membrane potentials or exists as a monomer in mitochondria with diminished membrane potential. Cells were seeded in 96-well plates at $1 \times 10^{4}$ cells/well. MMR was measured using a JC-1 mitochondrial membrane potential detection kit [21]. Before adding JC1 the medium was aspirated from the plates and adherent cells washed with PBS. The plates were incubated at $37^{\circ} \mathrm{C}$ for $20 \mathrm{~min}$ after the addition of $100 \mu \mathrm{L}$ of $1 \times \mathrm{JC}-1$ reagent into the wells. Cells were washed twice with PBS, and then PBS was added in an amount sufficient to cover the cell layer. Red fluorescence (excitation, $550 \mathrm{~nm}$, and emission, $600 \mathrm{~nm}$ ) and green fluorescence (excitation, $485 \mathrm{~nm}$, and emission, $535 \mathrm{~nm}$ ) were determined using a Softmax Pro fluorescence plate reader (Molecular Devices, Sunnyvale, CA, USA). The ratio of red-to-green fluorescence in dead cells and in cells undergoing apoptosis is decreased compared with healthy cells. For confocal microscopy, $1 \times$ of JC- 1 was added to treated cells for $15 \mathrm{~min}$ at $37^{\circ} \mathrm{C}$. Cells were imaged using Olympus FV10i-LIV confocal microscopes (Olympus, Tokyo, Japan). In live nonapoptotic cells, mitochondria appeared red due to aggregation of the JC-1 reagent. The red aggregates are excited at $559 \mathrm{~nm}$ and emit at 570-620 nm. In apoptotic and dead cells, the dye is monomeric and emits at 490-540 $\mathrm{nm}$ when excited at $473 \mathrm{~nm}$.

2.7. Mitochondrial Superoxide (MitoSOX). We used Mitotracker Red reagent for determining mitochondrial superoxide levels. Cells were plated at $1 \times 10^{4}$ cells on white plates for quantitating fluorescence, and $1 \times 10^{3}$ cells were added to cover slips for confocal analysis. The medium was removed, and cells were washed with PBS before measurements. Cells were incubated with $5 \mu \mathrm{M}$ MitoSOX Red for $20 \mathrm{~min}$ at $37^{\circ} \mathrm{C}$. MitoSOX Red has excitation/emission maxima of approximately $510 / 580 \mathrm{~nm}$. 


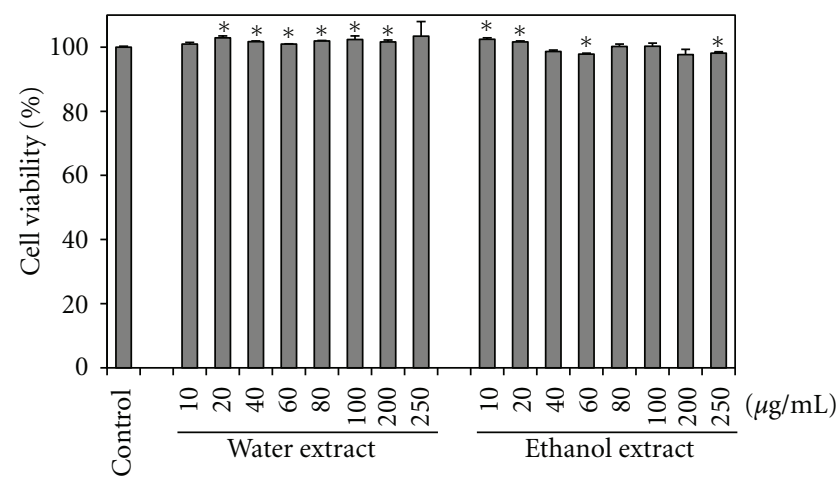

(a)

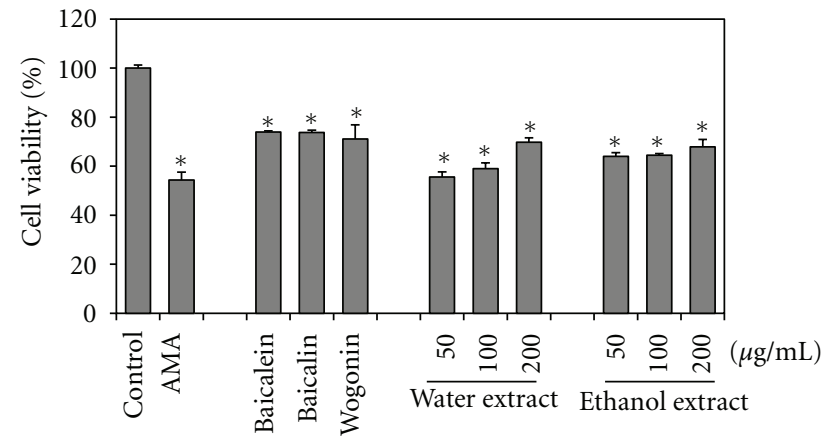

(b)

FIGURE 1: Effects of S. baicalensis extracts on cell viability and protective effects of flavonoids on L6 cells treated with AMA. (a) Cell viability of S. baicalensis extracts. (b) Cell-protective effect of samples treated with AMA. Cells were pretreated with baicalein, baicalin, and wogonin $(50 \mu \mathrm{g} / \mathrm{mL}$ each) for $1 \mathrm{~h}$ and pretreated with extracts at 50,100, $200 \mu \mathrm{g} / \mathrm{mL}$ each for $1 \mathrm{~h}$, and then $100 \mu \mathrm{g} / \mathrm{mL}$ of AMA was added.

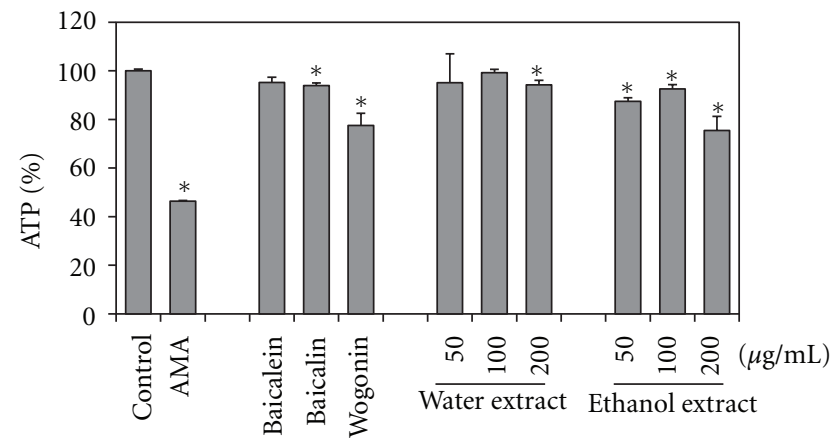

(a)

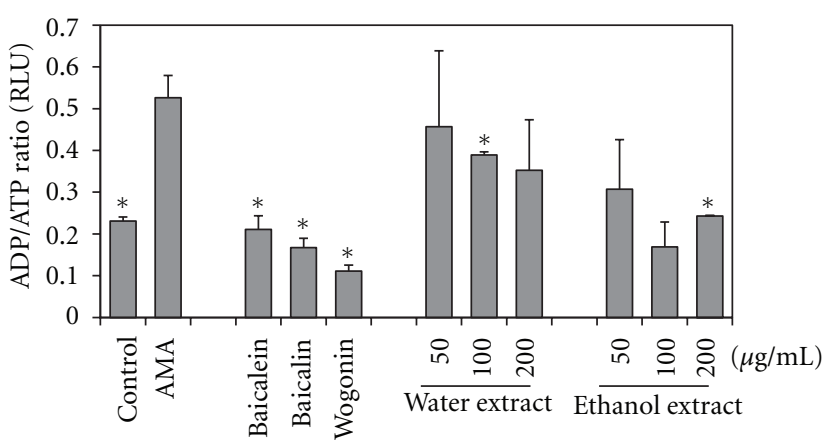

(b)

Figure 2: Effects of extracts on total cellular ATP levels and ADP/ATP ratio. (a) Total ATP levels in L6 cells treated with AMA. Cells were pretreated with baicalein, baicalin, or wogonin (each at $50 \mu \mathrm{g} / \mathrm{mL}$ ) for $1 \mathrm{~h}$. Cells were pretreated with extracts $(50,100,200 \mu \mathrm{g} / \mathrm{mL}$ each) for $1 \mathrm{~h}$, and then $100 \mu \mathrm{g} / \mathrm{mL}$ of AMA was added. ATP levels were measured using the ATP Lite luminescence-based assay in which results are reported as a percentage of the control. (b) ADP/ATP ratio in L6 cells was determined using the EnzyLight ADP/ATP ratio assay kit. Data were expressed as a percentage of control. ${ }^{*} P<0.05$.

2.8. Confocal Microscopy Analysis. Cells were plated at $1 \times$ $10^{3}$ cells on cover slips. The medium was removed, and cells were washed with PBS first. Cells were incubated for $45 \mathrm{~min}$ with $10 \mu \mathrm{M}$ Mitotracker Red and $1 \mu \mathrm{g} / \mathrm{mL}$ DAPI for detecting mitochondria. Cells were visualized by emission at $598 \mathrm{~nm}$ (excitation at $578 \mathrm{~nm}$ ). DAPI fluorescence was determined at an exciting wavelength of $359 \mathrm{~nm}$, and emission was detected at $461 \mathrm{~nm}$.

2.9. PGC-1 $\alpha$, SIRT1, and pAMPK Expression. Cells were incubated with varying concentrations of extracts and $100 \mu \mathrm{g} / \mathrm{mL}$ of AMA harvested and lysed in RIPA buffer (T\&I, Seoul, Korea). Protein concentrations were determined using the Protein Assay Reagent (Bio-Rad, Hercules, CA, USA). Proteins were separated by SDS-PAGE and transferred to PVDF membranes. The membrane was incubated with the primary antibody $(1: 1000)$ overnight and the secondary antibody $(1: 5000)$ for $2 \mathrm{~h}$. The blot was then developed using Luminol Enhancer solution (GE Healthcare, Waukesha, USA), visualized using an ImageQuant LAS 4000 mini
(GE Healthcare, Waukesha, USA), and quantified by using Image J densitometry software (Rasband, W.S., ImageJ, U. S. National Institutes of Health, Bethesda, Maryland, USA, http://imagej.nih.gov/ij/, 1997-2011).

2.10. Statistical Analyses. All data are expressed as mean \pm standard deviation of at least three independent experiments. Statistical analysis was performed using SPSS 20.0 (SPSS, Chicago, IL) by using one-way ANOVA followed by Tukey's post hoc test. Data were considered statistically significant at $P<0.05$.

\section{Results}

3.1. S. baicalensis Extracts and Flavonoids Protect against Antimycin A Toxicity. Cell viabilities were determined on L6 cells by MTS assay using water and $70 \%$ ethanol extracts from S. baicalensis. No cytotoxicity was observed in the cell from the extracts of S. baicalensis (Figure 1(a)). AMA toxicity significantly reflects the drug's damaging effects 


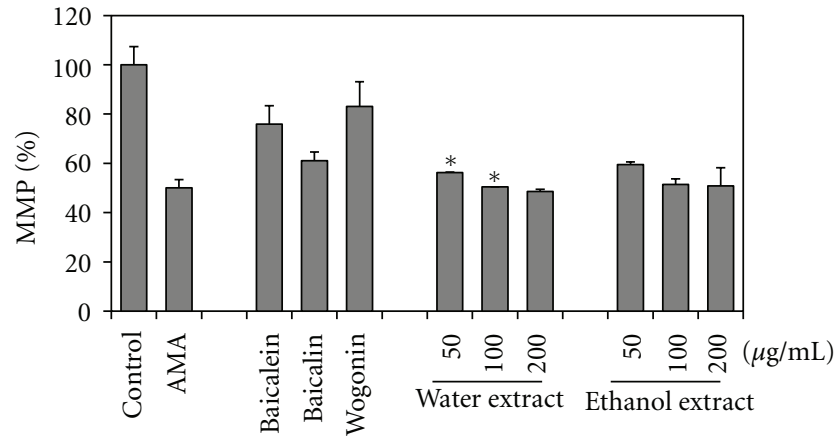

(a)

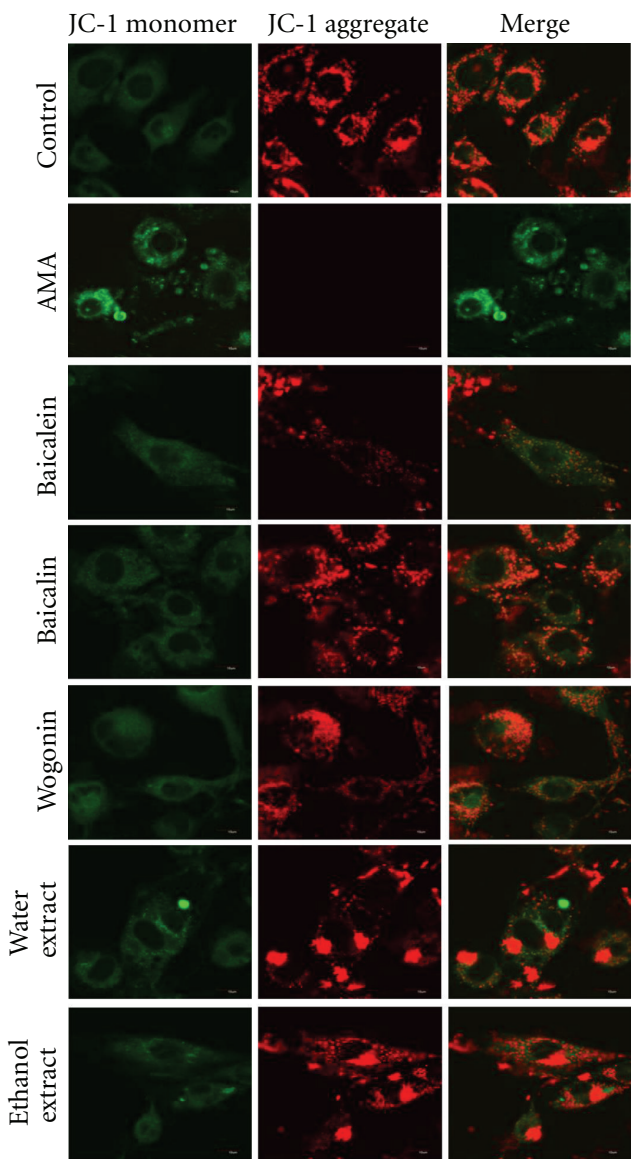

(b)

FIGURE 3: S. baicalensis extracts prevented polarized MMP. Cells were treated with AMA alone for $24 \mathrm{~h}$. (a) Cells were pretreated with baicalein, baicalin, wogonin $(50 \mu \mathrm{g} / \mathrm{mL}$ each) for $1 \mathrm{~h}$ and with extracts $(50,100,200 \mu \mathrm{g} / \mathrm{mL}$ each for $1 \mathrm{~h})$, and then $100 \mu \mathrm{g} / \mathrm{mL}$ of AMA was added. Red fluorescence (excitation $550 \mathrm{~nm}$, emission $600 \mathrm{~nm}$ ) and green fluorescence (excitation $485 \mathrm{~nm}$, emission $535 \mathrm{~nm}$ ) were determined by using a fluorescence plate reader. (b) Confocal images show JC-1 fluorescence $(60 \times 2.5)$. Cells were pretreated with $50 \mu \mathrm{g} / \mathrm{mL}$ for 1 , and then $100 \mu \mathrm{g} / \mathrm{mL}$ of AMA was added. Depolarized mitochondria were detected by green fluorescence, and polarized mitochondria were detected by red fluorescence. Data are expressed as a percentage of the control. $* P<0.05$.

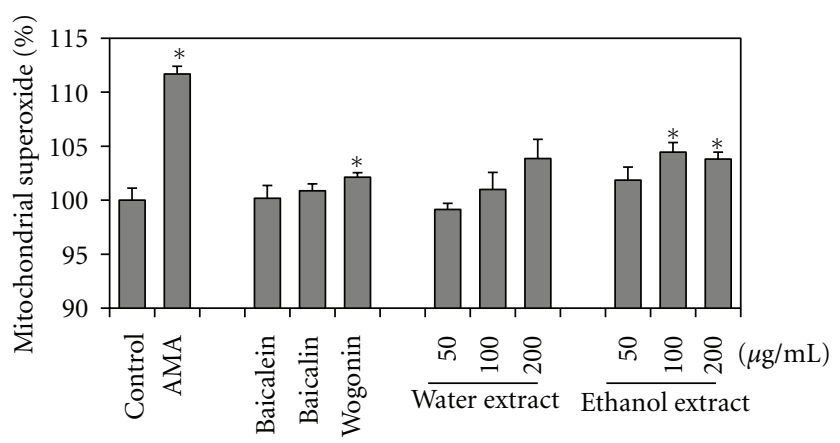

(a)
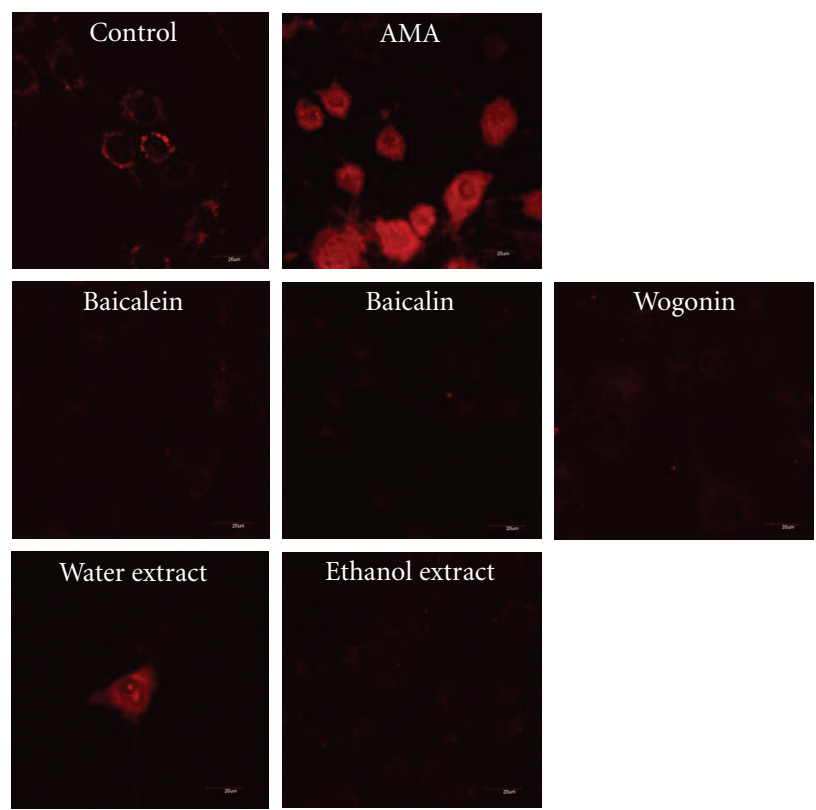

(b)

FIGURE 4: Determination of mitochondrial superoxide production measured using MitoSOX Red. (a) Cells were pretreated with baicalein, baicalin, wogonin $(50 \mu \mathrm{g} / \mathrm{mL}$ each $)$ for $1 \mathrm{~h}$ or with extracts $(50,100,200 \mu \mathrm{g} / \mathrm{mL}$ each) for $1 \mathrm{~h}$, and then $100 \mu \mathrm{g} / \mathrm{mL}$ AMA was added. (b) Determination of mitochondrial superoxide production measured by confocal microscopy $(60 \times 1.5)$. Samples were pretreated with $50 \mu \mathrm{g} / \mathrm{mL}$ for $1 \mathrm{~h}$, and then $100 \mu \mathrm{g} / \mathrm{mL}$ of AMA was added. Data were expressed as a percentage of control. ${ }^{*} P<$ 0.05 .

on mitochondria. We then determined the dose and time of exposure to $100 \mu \mathrm{g} / \mathrm{mL}$ AMA required to reduce cell viability by $50 \%$ after $24 \mathrm{~h}$ incubation (data not shown). Cells were then treated with $100 \mu \mathrm{g} / \mathrm{mL}$ AMA in the presence of $S$. baicalensis extracts or individual flavonoids. Baicalein, baicalin, and wogonin significantly increased cell viability by $20 \%$ compared with AMA alone (Figure 1(b)). Here all flavonoids performed almost the same for cell viability. Cell viability was increased by addition of $50 \mu \mathrm{g} / \mathrm{mL}$ to $200 \mu \mathrm{g} / \mathrm{mL}$ of water and $70 \%$ ethanol showing slightly lower compared to flavonoids, and within the extracts treatment, water extracts performed slightly higher for cell viability compared to ethanol extracts treatment. 
3.2. Effects of S. baicalensis Extracts and Flavonoids on ATP, $A D P$ Levels. To determine whether the extracts or flavonoids affected energy production, we measured ATP levels. ATP production decreased in the AMA-treated group but increased significantly in cells treated with flavonoids and extracts of $S$. baicalensis (Figure 2(a)). ATP production increased by $50 \%$ in cells treated with flavonoids (except wogonin) and extracts of S. baicalensis compared to AMAtreated group (Figure 2(a)). Among the treatments water extract at $100 \mu \mathrm{g} / \mathrm{mL}$ yielded the highest ATP production (Figure 2(a)). The ADP/ATP ratio was substantially elevated in AMA-treated cells compared with controls (Figure 2(b)). The three flavonoids decreased the ADP/ATP ratios, by wogonin in particular. The ethanol extracts reduced this ratio to a great extent.

3.3. Mitochondrial ROS Production. Disruption of MMP results from mitochondria dysfunction and induction of apoptosis. AMA depolarizes mitochondrial membrane. To study the direct effect of $S$. baicalensis extracts on AMAinduced oxidative stress, we determined MMP. Cells were incubated with flavonoids and extracts of S. baicalensis for $1 \mathrm{~h}$, and then $100 \mu \mathrm{g} / \mathrm{mL}$ of AMA was added and cells incubated for $24 \mathrm{~h}$. The red AMA signal decreased greatly, and the red/green signal ratio also decreased using JC-1 (Figure 3(a)). Cells in control treatment have both green and red signal, showing mitochondria exactly (Figure 3(b)). AMA treatment for $24 \mathrm{~h}$ caused a significant reduction in JC1 ratio indicating a depolarized MMP, whereas flavonoids and extracts treatment induced a significant increase in MMP as follows: by $83.1 \%, 75.9 \%, 61.1 \%, 59.5 \%$, and $56.3 \%$ using wogonin, baicalein, baicalin, ethanol extracts, and water extracts, respectively (Figure 3(a)) as indicated by an increase in red JC-1 fluorescence (Figure 3(b)).

3.4. Changes of Mitochondrial Superoxide Levels. Quantitative measurements of the mean intensity from the AMAinduced cells demonstrated a $111.7 \%$ increase compared with control cells (Figure 4(a)). In contrast, mitochondrial superoxide levels decreased around $10 \%$ when cells were treated with flavonoids and extracts. Confocal microscope imaging demonstrated an increase in mitochondrial MitoSOX fluorescence in cells treated with AMA for $24 \mathrm{~h}$ (Figure 4(b)). The mitochondria of AMA-induced cells exhibited red fluorescence, indicating the presence of superoxide, whereas the control, flavonoids, and extracts of $S$. baicalensis-treated cells did not show or showed slight red fluorescence indicating a low level of superoxide.

3.5. Confocal Analysis of Mitochondria. Mitotracker probes can be used to stain the mitochondria of L6 cells with red fluorescence. Confocal observations revealed evenly distributed mitochondrial staining in control cells (Figure 5). AMAinduced cells showed reduced red fluorescence intensity in mitochondria indicating a depolarization of the inner mitochondrial membrane. The mitochondrial staining pattern was restored from that of control cells by $1 \mathrm{~h}$ treatment with $50 \mu \mathrm{g} / \mathrm{mL}$ of baicalein, baicalin, and wogonin. Extracts

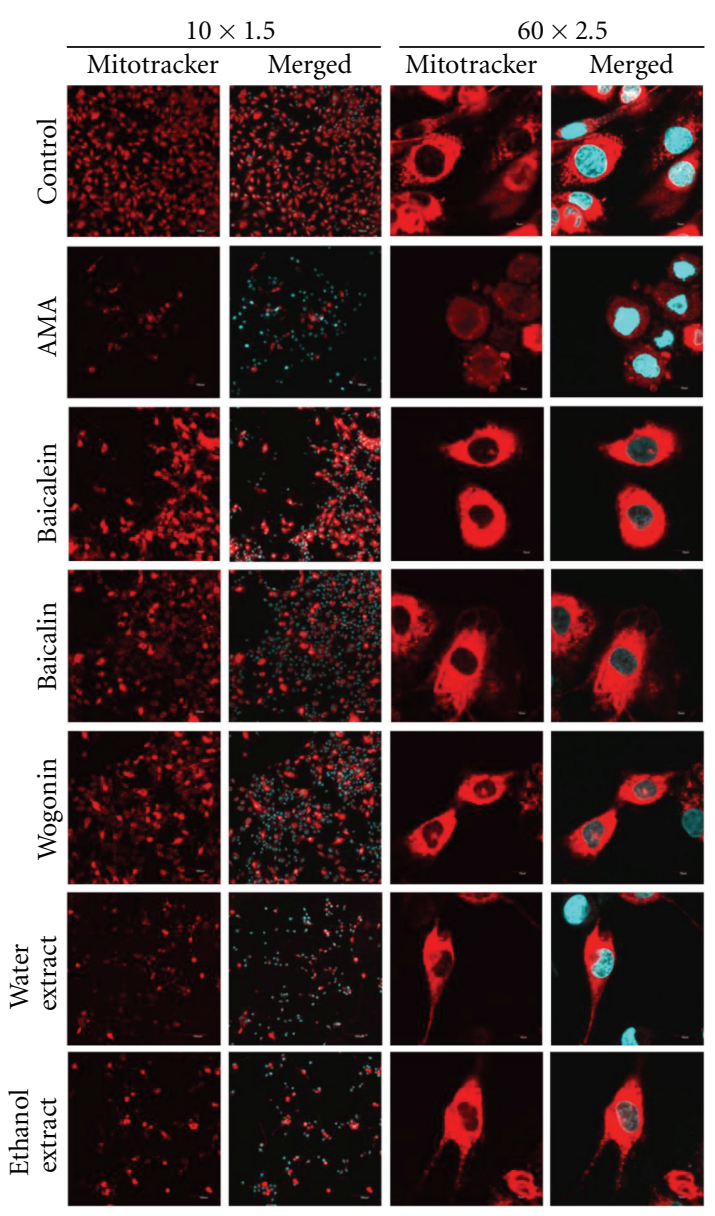

FIGURE 5: Confocal microscopy of mitochondria. Distribution of Mitotracker red fluorescence indicated mitochondria, and merged images indicated colocalization with DAPI blue fluorescence $(10 \times$ 1.5 and $60 \times 2.5)$. Samples were treated with $50 \mu \mathrm{g} / \mathrm{mL}$ for $1 \mathrm{~h}$, and before $100 \mu \mathrm{g} / \mathrm{mL}$ of AMA were added.

treatment also increased the red fluorescence intensity and membrane permeability to DAPI.

3.6. PGC-1 $\alpha$, SIRT1, and pAMPK Expression. PGC- $1 \alpha$, SIRT1, and pAMPK were expressed in L6 cells as determined by Western blotting analysis (Figure 6). The expression of each protein was increased by treatment of cells with flavonoids or extracts of S. baicalensis-treated cells compared to AMA-treated cells. Among the treatments of flavonoids and extracts, water extracts performed the best for expressing the intensity of protein. PGC- $1 \alpha$ levels increased when cells were treated with flavonoids and extracts of $S$. baicalensis. In particular, the water extracts and baicalin at $50 \mu \mathrm{g} / \mathrm{mL}$ increased PGC- $1 \alpha$ by 47.7 and $40 \%$, respectively. SIRT1 intensity increased by $30 \%$ and $19 \%$ using more water and ethanol extracts of $S$. baicalensis at $50 \mu \mathrm{g} / \mathrm{mL}$, respectively, compared with AMA cells. All the treated cells with flavonoids and extracts exhibited higher pAMPK intensity compared with AMA cells. Water extract and ethanol extracts at $50 \mu \mathrm{g} / \mathrm{mL}$ increased the intensity of pAMPK by 36.6 and 20.2\%, respectively, compared with AMA cells. 


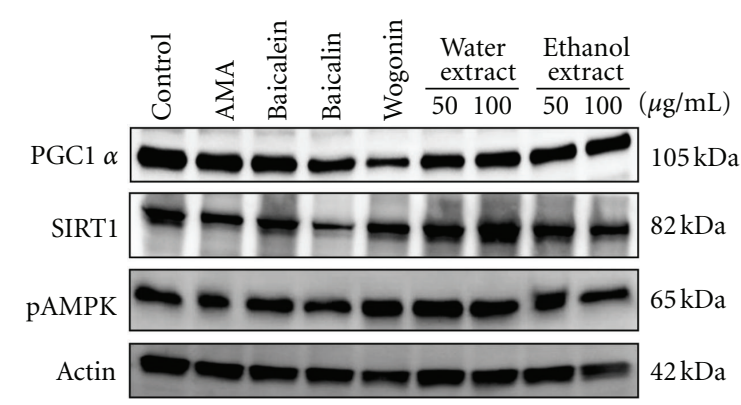

(a)

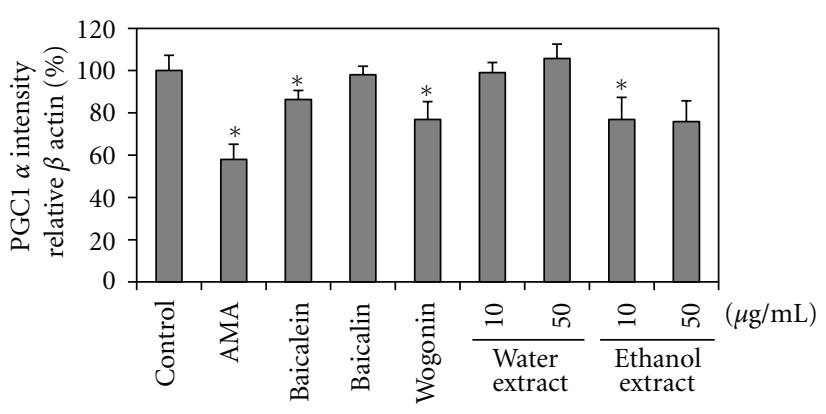

(c)

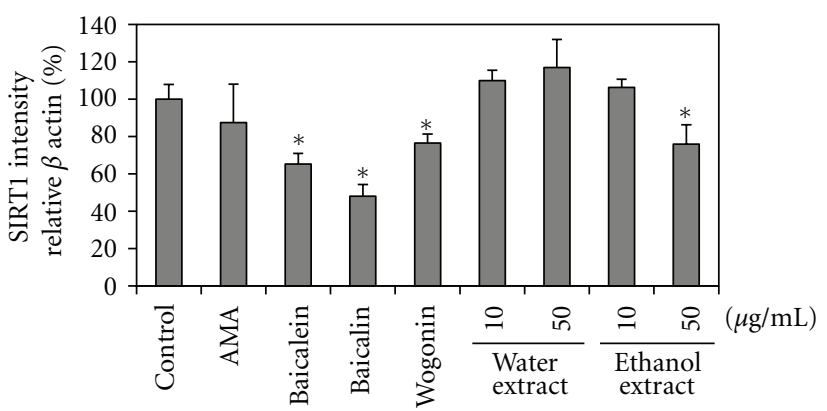

(b)

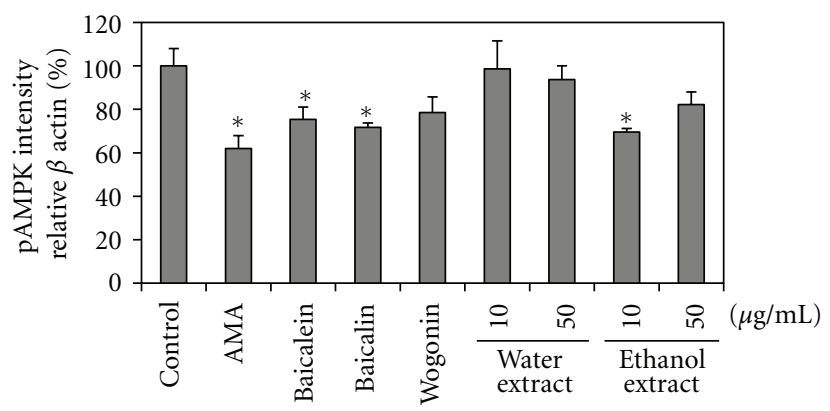

(d)

FIGURE 6: PGC-1 $\alpha$, SIRT1, and pAMPK protein expression analysis in L6 cells. Cell lysates were prepared from L6 cells, were pretreated with extracts for $1 \mathrm{~h}$, and then exposed to AMA. The phosphorylated and total protein levels were detected with specific antibodies by Western blotting. $\beta$-actin served as the loading control. Quantitative analysis was performed by measuring the signal intensity relative to the control $(n=3)$.

\section{Discussion}

In the present study, we focused on the flavonoids and extracts prepared from $S$. baicalensis for their effect on mitochondrial dysfunction (as indicated by oxidative stress) induced by AMA. Cell viability was increased by the addition of baicalein, baicalin, and wogonin before. These results agree with the findings of others that apocynin protects against AMA-induced cell damage in osteoblastic MC3T3-E1 cells [22]. Mitochondrial dysfunction induced by AMA results in decreased ATP production. Oxidative stress increases respiration and generation of ROS, resulting in ATP depletion [23]. We show here that treatment with baicalein, baicalin, and wogonin before AMA exposure significantly prevented loss of ATP and MMP, suggesting that molecules present in $S$. baicalensis protect cells from mitochondrial dysfunction. Thus, the percentage of extracellular ATP in AMA treated cells was $46.36 \%$, but pretreating samples with flavonoids and extracts of $S$. baicalensis increased this value.

Mitochondrial ROS production is intimately linked to MMP, such that hyperpolarization increases and promotes ROS production [24]. Our data presented here show that cells treated with $S$. baicalensis extracts exhibited decreased MMP and increased mitochondrial biogenesis. The disruption of MMP in L6 cells is the result of ROS-mediated damage. The MitoSOX Red results indicate that mitochondrial superoxide was increased in AMA-induced cells.
As the major producer and primary target of ROS, mitochondria play an important role in aging. Our present study demonstrates that cells treated with baicalein, baicalin, and wogonin prevented AMA-induced ROS production by mitochondria. Mitotracker Red dyes are being used with increasing frequency for morphological and functional measurements of mitochondria. Here, Mitotracker Red fluorescence delocalized during mitochondrial depolarization and increased following treatment with AMA, indicating a decrease in the capacity of these cells to generate energyrich reductants. Baicalein, baicalin, and wogonin treatment significantly increased mitochondrial number compared with AMA-induced cells.

PGC- $1 \alpha$, SIRT1, and pAMPK have been identified as the major transcription factors controlling expression of mitochondrial genes; however, the signaling pathways between the mitochondria and the nucleus remain to be elucidated [4]. SIRT1 regulates aging and resistance to oxidative stress in the heart in vivo, and stimulation of SIRT1 may be considered as an antiaging therapy for the heart [25]. An SIRT1 activator, resveratrol, induces PGC- $1 \alpha$ activity by facilitating SIRT1mediated deacetylation [26]. SIRT1 is activated and cooperates with pAMPK to enhance the ability of PGC- $1 \alpha$ to stimulate mitochondrial biogenesis and function [27]. AMPK directly phosphorylates PGC- $1 \alpha$, enhancing its activity at its own promoter and triggering a transcriptional cascade that increases expression of PGC- $1 \alpha$ and its target genes 
that are involved in mitochondrial biogenesis [28]. Here, we found that L6 cells treated with flavonoids and extracts of $S$. baicalensis upregulated SIRT1 levels and stimulated pAMPK activity in AMA-induced cells. Activation of AMPK increases the activities of citrate synthase and succinate dehydrogenase, which may regulate mitochondrial biogenesis in response to energy depletion. AMPK phosphorylates PGC- $1 \alpha$, a key regulator of mitochondrial biogenesis and metabolism [29]. Quercetin protects against $\mathrm{H}_{2} \mathrm{O}_{2}$-induced cell death and increases mitochondrial biogenesis by upregulating PGC- $1 \alpha$ and SIRT 1, which regulate mitochondrial activity in human retinal pigment epithelium in vitro [30]. Mitochondrial dysfunction, characterized by a decline in cellular ATP, loss of MMP, and decrease of protein content, is central to the execution of cell death.

\section{Conclusion}

In conclusion, the protection of mitochondrial dysfunction by S. baicalensis extracts (containing the flavonoids baicalein, baicalin, and wogonin) especially water extracts from AMAinduced oxidative damage and cell death can be attributed to increased ATP levels, regulation of MMP, and increased mitochondrial function mediated by PGC- $1 \alpha$, SIRT1, and pAMPK.

\section{Acknowledgment}

This work was supported by a grant from the Korea Institute of Oriental Medicine (KIOM).

\section{References}

[1] Y. S. Ho, K. F. So, and R. C. C. Chang, "Anti-aging herbal medicine-how and why can they be used in aging-associated neurodegenerative diseases?" Ageing Research Reviews, vol. 9, no. 3, pp. 354-362, 2010.

[2] D. J. Bonda, H. G. Lee, A. Camins et al., "The sirtuin pathway in ageing and Alzheimer disease: mechanistic and therapeutic considerations," The Lancet Neurology, vol. 10, no. 3, pp. 275279, 2011.

[3] H. Cui, Y. Kong, and H. Zhang, "Oxidative stress, mitochondrial dysfunction, and aging," Journal of Signal Transduction, vol. 2012, Article ID 646354, 13 pages, 2012.

[4] L. W. S. Finley and M. C. Haigis, "The coordination of nuclear and mitochondrial communication during aging and calorie restriction," Ageing Research Reviews, vol. 8, no. 3, pp. 173$188,2009$.

[5] C. Mammucari and R. Rizzuto, "Signaling pathways in mitochondrial dysfunction and aging," Mechanisms of Ageing and Development, vol. 131, no. 7-8, pp. 536-543, 2010.

[6] Y. H. Han, S. H. Kim, S. Z. Kim, and W. H. Park, "Antimycin $\mathrm{A}$ as a mitochondria damage agent induces an $\mathrm{S}$ phase arrest of the cell cycle in HeLa cells," Life Sciences, vol. 83, no. 9-10, pp. 346-355, 2008.

[7] Y. H. Han, S. H. Kim, S. Z. Kim, and W. H. Park, "Antimycin A as a mitochondrial electron transport inhibitor prevents the growth of human lung cancer A549 cells," Oncology Reports, vol. 20, no. 3, pp. 689-693, 2008.
[8] C. Piskernik, S. Haindl, T. Behling et al., "Antimycin A and lipopolysaccharide cause the leakage of superoxide radicals from rat liver mitochondria," Biochimica et Biophysica Acta, vol. 1782, no. 4, pp. 280-285, 2008.

[9] W. H. Huang, A. R. Lee, and C. H. Yang, "Antioxidative and anti-inflammatory activities of polyhydroxyflavonoids of Scutellaria baicalensis GEORGI," Bioscience, Biotechnology and Biochemistry, vol. 70, no. 10, pp. 2371-2380, 2006.

[10] Y. Zhang, X. Wang, X. Wang et al., "Protective effect of flavonoids from Scutellaria baicalensis Georgi on cerebral ischemia injury," Journal of Ethnopharmacology, vol. 108, no. 3, pp. 355-360, 2006.

[11] S. Su, C. M. He, L. C. Li, J. K. Chen, and T. S. Zhou, "Genetic characterization and phytochemical analysis of wild and cultivated populations of Scutellaria baicalensis," Chemistry and Biodiversity, vol. 5, no. 7, pp. 1353-1363, 2008.

[12] S. B. Yoon, Y. J. Lee, S. K. Park et al., "Anti-inflammatory effects of Scutellaria baicalensis water extract on LPS-activated RAW 264.7 macrophages," Journal of Ethnopharmacology, vol. 125, no. 2, pp. 286-290, 2009.

[13] E. H. Kim, B. Shim, S. Kang et al., "Anti-inflammatory effects of Scutellaria baicalensis extract via suppression of immune modulators and MAP kinase signaling molecules," Journal of Ethnopharmacology, vol. 126, no. 2, pp. 320-331, 2009.

[14] K. Jeong, Y. C. Shin, S. Park et al., "Ethanol extract of Scutellaria baicalensis Georgi prevents oxidative damage and neuroinflammation and memorial impairments in artificial senescense mice," Journal of Biomedical Science, vol. 18, no. 1, p. 14, 2011.

[15] X. W. Zhang, W. F. Li, W. W. Li et al., "Protective effects of the aqueous extract of Scutellaria baicalensis against acroleininduced oxidative stress in cultured human umbilical vein endothelial cells," Pharmaceutical Biology, vol. 49, no. 3, pp. 256-261, 2011.

[16] W. H. Chang, C. H. Chen, and F. J. Lu, "Different effects of baicalein, baicalin and wogonin on mitochondrial function, glutathione content and cell cycle progression in human hepatoma cell lines," Planta Medica, vol. 68, no. 2, pp. 128132, 2002.

[17] Z. Gao, K. Huang, and H. Xu, "Protective effects of flavonoids in the roots of Scutellaria baicalensis Georgi against hydrogen peroxide-induced oxidative stress in HS-SY5Y cells," Pharmacological Research, vol. 43, no. 2, pp. 173-178, 2001.

[18] I. K. Lee, K. A. Kang, R. Zhang, B. J. Kim, S. S. Kang, and J. W. Hyun, "Mitochondria protection of baicalein against oxidative damage via induction of manganese superoxide dismutase," Environmental Toxicology and Pharmacology, vol. 31, no. 1, pp. 233-241, 2011.

[19] S. Ueda, H. Nakamura, H. Masutani et al., "Baicalin induces apoptosis via mitochondrial pathway as prooxidant," Molecular Immunology, vol. 38, no. 10, pp. 781-791, 2002.

[20] R. D. Petty, L. A. Sutherland, E. M. Hunter, and I. A. Cree, "Comparison of MTT and ATP-based assays for the measurement of viable cell number," Journal of Bioluminescence and Chemiluminescence, vol. 10, no. 1, pp. 29-34, 1995.

[21] G. Yao, L. Ling, J. Luan, D. Ye, and P. Zhu, "Nonylphenol induces apoptosis of Jurkat cells by a caspase- 8 dependent mechanism," International Immunopharmacology, vol. 7, no. 4, pp. 444-453, 2007.

[22] E. M. Choi and Y. S. Lee, "Protective effect of apocynin on antimycin A-induced cell damage in osteoblastic MC3T3-E1 cells," Journal of Applied Toxicology, 2011. 
[23] B. S. Tiwari, B. Belenghi, and A. Levine, "Oxidative stress increased respiration and generation of reactive oxygen species, resulting in ATP depletion, opening of mitochondrial permeability transition, and programmed cell death," Plant Physiology, vol. 128, no. 4, pp. 1271-1281, 2002.

[24] M. Valko, D. Leibfritz, J. Moncol, M. T. D. Cronin, M. Mazur, and J. Telser, "Free radicals and antioxidants in normal physiological functions and human disease," International Journal of Biochemistry and Cell Biology, vol. 39, no. 1, pp. 4484, 2007.

[25] R. A. Ralph, G. Shymin, Z. Peiyong et al., "Sirt1 regulates aging and resistance to oxidative stress in the heart," Circulation Research, vol. 100, no. 10, pp. 1512-1521, 2007.

[26] M. Lagouge, C. Argmann, Z. Gerhart-Hines et al., "Resveratrol improves mitochondrial function and protects against metabolic disease by activating SIRT1 and PGC-1 $\alpha$," Cell, vol. 127, no. 6, pp. 1109-1122, 2006.

[27] N. B. Ruderman, X. J. Xu, L. Nelson et al., "AMPK and SIRT1: a long-standing partnership?" American Journal of PhysiologyEndocrinology and Metabolism, vol. 298, no. 4, pp. E751-E760, 2010.

[28] S. Jäer, C. Handschin, J. St-Pierre, and B. M. Spiegelman, "AMP-activated protein kinase (AMPK) action in skeletal muscle via direct phosphorylation of PGC-1 $\alpha$," Proceedings of the National Academy of Sciences of the United States of America, vol. 104, no. 29, pp. 12017-12022, 2007.

[29] M. R. Richard and I. S. Gerald, "The role of AMP-activated protein kinase in mitochondrial biogenesis," Journal of Physiology, vol. 574, no. 1, pp. 33-39, 2006.

[30] D. Kook, A. H. Wolf, A. L. Yu et al., "The protective effect of quercetin against oxidative stress in the human RPE in vitro," Investigative Ophthalmology and Visual Science, vol. 49, no. 4, pp. 1712-1720, 2008. 


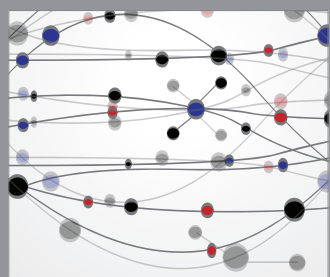

The Scientific World Journal
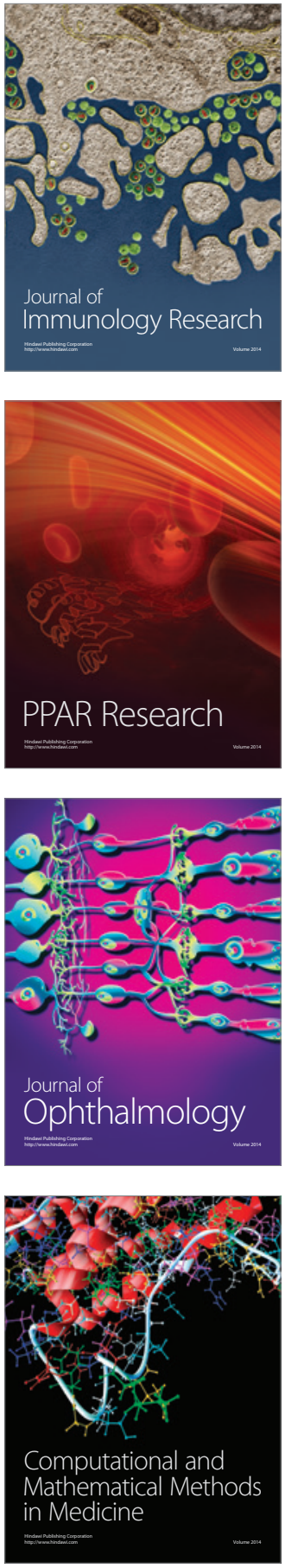

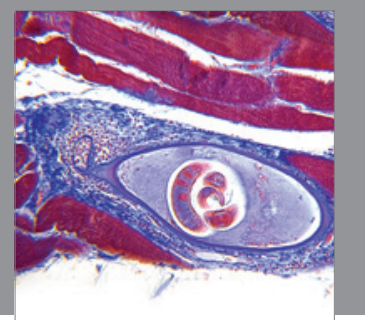

Gastroenterology

Research and Practice
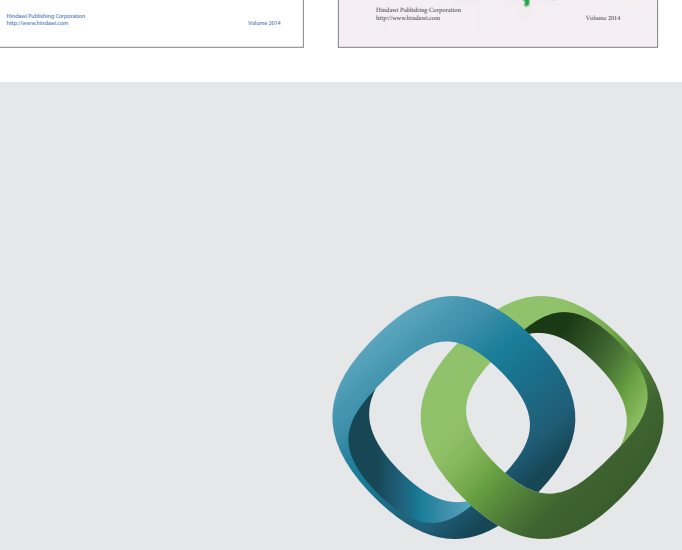

\section{Hindawi}

Submit your manuscripts at

http://www.hindawi.com
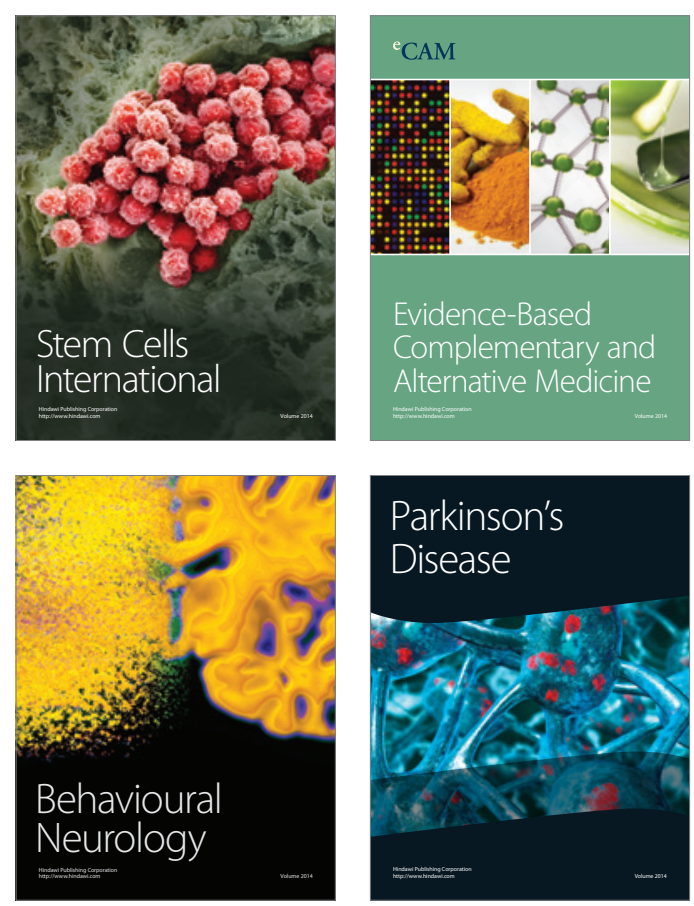

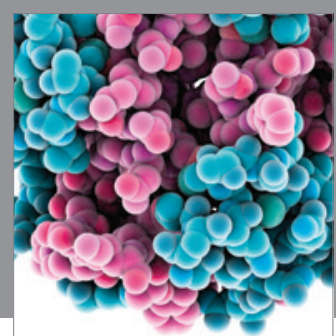

Journal of
Diabetes Research

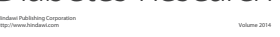

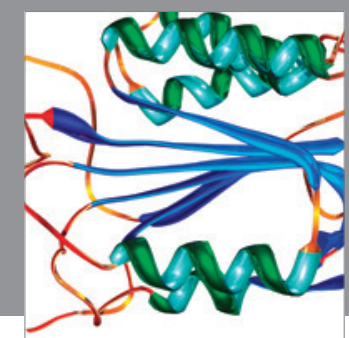

Disease Markers
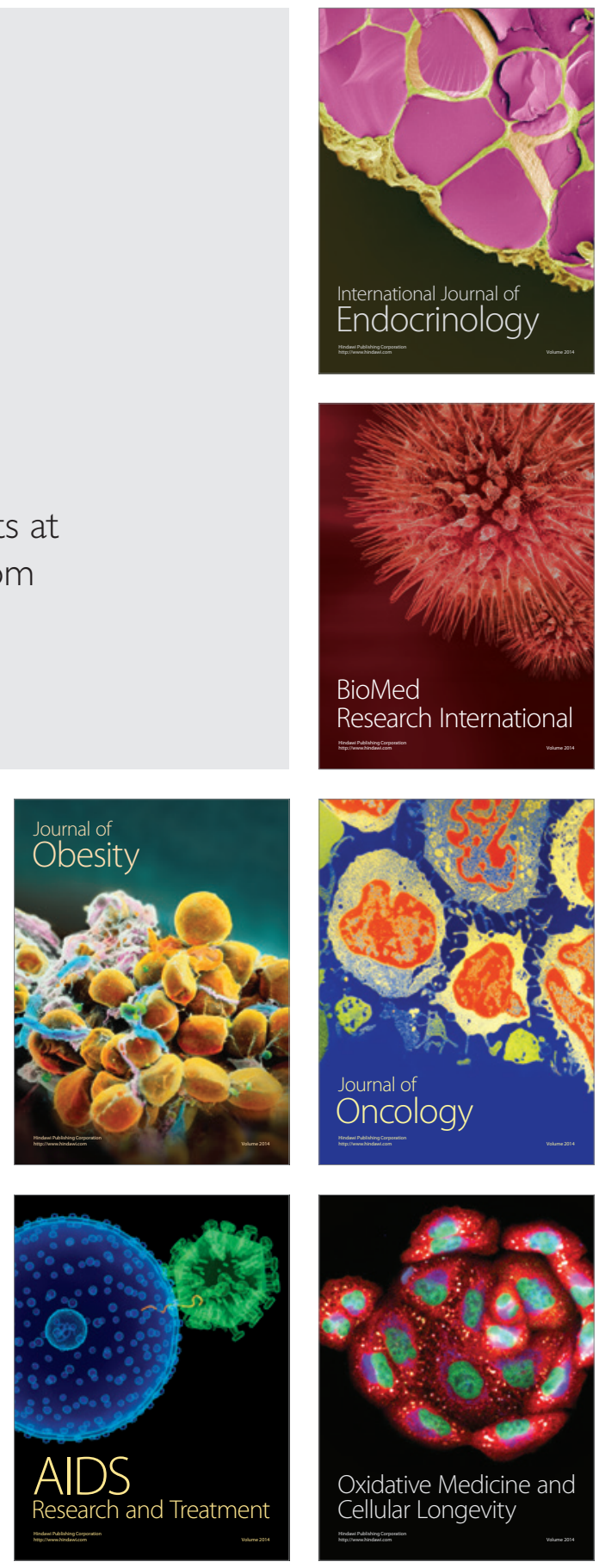